\title{
Molecular detection of Culicoides spp. and Culicoides imicola, the principal vector of bluetongue (BT) and African horse sickness (AHS) in Africa and Europe
}

\author{
Catherine CÊTRE-SOSSAH ${ }^{\mathrm{a} *}$, Thierry BALDET ${ }^{\mathrm{a}}$, Jean-Claude DELÉCOLLE ${ }^{\mathrm{b}}$, \\ Bruno MATHIEU ${ }^{\mathrm{c}}$, Aurélie PERRIN ${ }^{\mathrm{a}}$, Colette GRILLET ${ }^{\mathrm{a}}$, Emmanuel ALBINA $^{\mathrm{a}}$ \\ ${ }^{a}$ CIRAD-EMVT, Campus international de Baillarguet, TA30/G, 34398 Montpellier Cedex 5, France \\ b Université Louis Pasteur de Strasbourg, Musée zoologique, 29 bd de la Victoire, \\ 67000 Strasbourg, France \\ ${ }^{c}$ Entente Interdépartementale pour la Démoustication, 165 avenue Paul Rimbaud, \\ 34184 Montpellier Cedex 4, France
}

(Received 12 November 2003; accepted 9 February 2004)

\begin{abstract}
Bluetongue (BT) and African Horse Sickness (AHS) are infectious arthropod-borne viral diseases affecting ruminants and horses, respectively. Culicoides imicola Kieffer, 1913, a biting midge, is the principal vector of these livestock diseases in Africa and Europe. Recently bluetongue disease has re-emerged in the Mediterranean Basin and has had a devastating effect on the sheep industry in Italy and on the islands of Sicily, Sardinia, Corsica and the Balearics, but fortunately, has not penetrated onto mainland France and Spain. To survey for the presence of $C$. imicola, an extensive light-trap network for the collection of Culicoides, was implemented in 2002 in southern mainland France. The morphological identification of Culicoides can be both tedious and time-consuming because its size ranges from 1.5 to $3 \mathrm{~mm}$. Therefore, an ITS $_{1} \mathrm{rDNA}$ polymerase chain reaction (PCR)-based diagnostic assay was developed to rapidly and reliably identify Culicoides spp. and C. imicola. The aim of this work was to set up a rapid test for the detection of $C$. imicola amongst a pool of insects collected in areas at risk for BT. The sequence similarity of the rDNA (nuclear ribosomal DNA), which is greater within species than between species, is the foundation of its utilisation in species-diagnostic assays. The alignment of the 11 ITS1 sequences of Culicoides obtained from Genbank and EMBL databases helped us to identify one region in the 5' end and one in the 3' end that appear highly conserved. PCR primers were designed within these regions to amplify genus-specific fragments. In order to set up a C. imicola-specific PCR, another forward primer was designed and used in combination with the previously designed reverse primer. These primers proved to be highly specific and sensitive and permitted a rapid diagnostic separation of $C$. imicola from Culicoides spp.
\end{abstract}

molecular detection / Culicoides imicola / ITS1 / PCR / bluetongue

\section{INTRODUCTION}

Bluetongue (BT) and African horse sickness (AHS) are infectious arthropod-borne viral diseases affecting ruminants and horses, respectively. Bluetongue disease is caused by the bluetongue virus (BTV), which is the prototype of the Orbivirus

* Corresponding author: catherine.cetre-sossah@cirad.fr 
genus within the Reoviridae family [11]. AHSV is another member of this family and genus. Both viruses are transmitted by certain species of biting midges belonging to the Culicoides genus (Diptera: Ceratopogonidae) and are maintained in nature through a series of alternating cycles of replication between its Culicoides vectors and susceptible hosts [25].

The spread of bluetongue across all continents pantropically (located between $44^{\circ} \mathrm{N}$ and $35^{\circ} \mathrm{S}$ ) depends mainly upon the distribution and seasonal abundance of the insect vectors. More than 1200 Culicoides species have been identified in the world [3] but only 17 have been connected with BTV transmission [18]. The major vector species are C. imicola and C. bolitinos in Africa, C. imicola in Asia, C. fulvus and C. brevitarsis in Australia, C. sonorensis in North America, C. insignis and C. pusillus in South and Central America [17, 18].

As with bluetongue, AHSV is limited to geographical areas where the vector $C$. imicola is present, and its spread depends on the occurrence of climatic conditions favouring vector activity. AHSV is endemic in subSaharan Africa but periodically makes brief excursions beyond this area, where it has caused major epizootics extending as far as Pakistan and India in the east, and as far as Morocco, Spain and Portugal in the west [4, $20,21,23]$. More than 300000 equines died during the great epizootic of 1959-1961 in the Middle-East and South-West Asia [12]. Until recently, the virus had not survived across more than two seasons in any of these epizootic areas. However, the recent outbreak of AHSV in the western Mediterranean basin, which lasted for five years (1987-1991), has forced us to re-assess the situation with greater vigilance.

The explosive outbreaks of BTV in the Mediterranean Basin since 1998 have been fuelled largely by the classical Afro-Asian vector $C$. imicola $[1,19]$. It is known that C. imicola s.l. is a complex of at least $10 \mathrm{sib}-$ ling species but for the time being only C. imicola s.s. is present in Europe [16]. In the eastern Mediterranean Basin, outbreaks of BTV have occurred in Bulgaria, Serbia, Kosovo, Croatia, Montenegro, northern Greece and Bosnia Herzegovina in regions up to $44^{\circ} 30^{\prime} \mathrm{N}$, which is further north within Europe than has ever been seen before, and where $C$. imicola has not been detected during insect surveys $[2,19]$. This strongly suggests that here the virus is being transmitted by other vector species, possibly by $C$. pulicaris and one or more species of the C. obsoletus complex. These Palaearctic species or species complexes are widespread and abundant in the region and across most of northern Europe.

As a consequence of the discovery of C. imicola on the island of Corsica in October 2000 [7], the subsequent BTV serotype 2 outbreaks in the autumn of 2000 and 2001 [29-31], and the relatively high risk of occurrence of this vector in neighbouring coastal mainland in southern France [28], it was decided to study the Culicoides fauna of this region. For this purpose, a large lighttrap network was implemented in 2002 in southern mainland France to survey for the possible presence of $C$. imicola. The morphological identification of Culicoides can be both tedious and time consuming because its size ranges from 1.5 to $3 \mathrm{~mm}$. Thus the aim of this study was to set up a rapid diagnostic test for the detection of $C$. imicola amongst a pool of insects collected in areas where livestock are thought to be at risk for BT. The study of molecular taxonomy and phylogenetic evolution of this genus should also give interesting results.

Nuclear ribosomal DNA (rDNA) has several unique features which make it an ideal DNA target for systematic studies [6]. It consists of tandemly repeated copies of the transcriptional unit with three gene regions (18S, 5.8S and 28S) separated by transcribed spacers [8]. As shown in Figure 1, the external transcribed spacer (ETS) is located upstream of the $18 \mathrm{~S}$ gene, with the first $\left(\operatorname{ITS}_{1}\right)$, and second $\left(\right.$ ITS $\left._{2}\right)$, internal transcribed spacers (ITS) located between either the genes $18 \mathrm{~S}$ and $5.8 \mathrm{~S}$, or between 


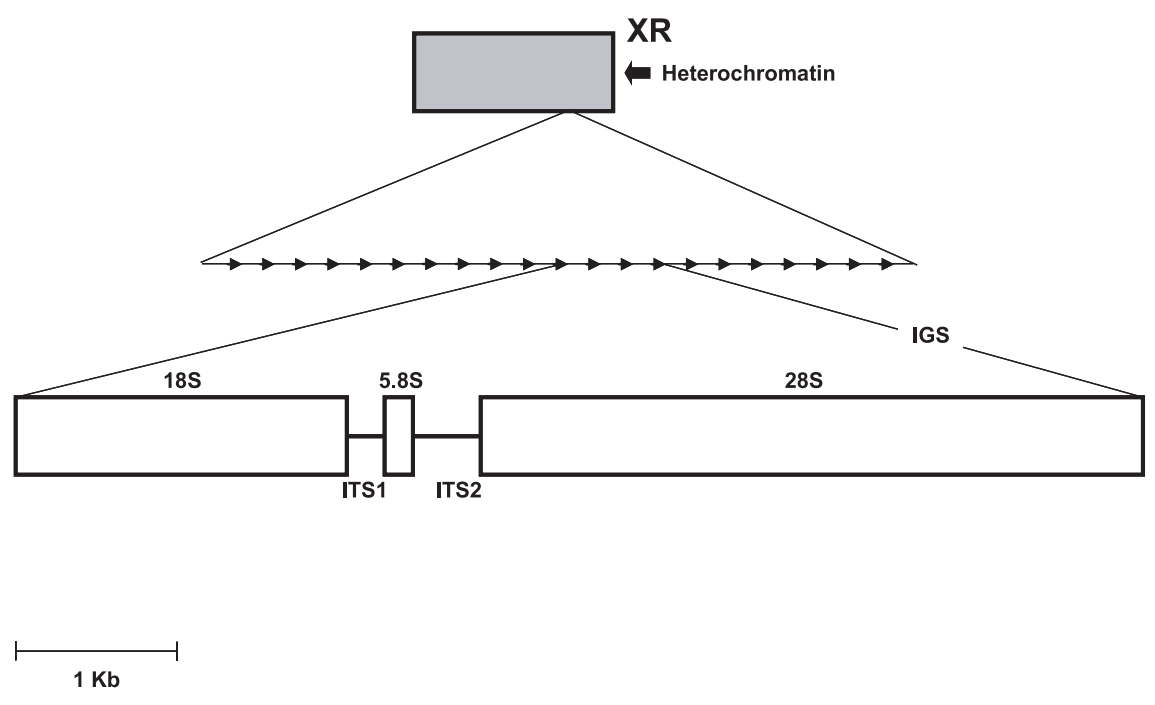

Figure 1. Location and organisation of the ribosomal DNA (rDNA). The rDNA locus consists of one very long array of repeat units (500-700 repeats per genome). Each repeat is $9 \mathrm{~kb}$ long and consists of the genes for the $18 \mathrm{~S}, 5.8 \mathrm{~S}$, and $28 \mathrm{~S}$ rDNA (open rectangles). These genes are separated by spacers (solid lines), the internal transcribed spacers (ITS1 and ITS2), and the intergenic spacer (IGS). Adapted from [10].

5.8S and 28S, respectively. It contains regions of varying evolutionary rates, from highly conserved regions (e.g. 5.8S and 18S rRNA genes) to more variable ones (e.g. spacers). Sequence similarity of the rDNA, which is greater within than between species, is the foundation of its utilisation for species-diagnostic assays. A recent study using internal transcribed spacers (ITS) of nuclear ribosomal DNA from Culicoides has been performed for a phylogenetic analysis of the genus [15]. Previously, rDNA genetic markers have been used for the identification of mosquitoes (Anopheles gambiae, An. nili and An. minimus using ITS) $[9,10,13,22$, 26]. The alignment of the 11 ITS 1 sequences of Culicoides obtained from Genbank and EMBL databases helped us to focus on one region in the 5' end and one in the 3 ' end appeared highly conserved. Within these regions, PCR primers were designed to amplify genus-specific fragments. In order to set up a $C$. imicola-specific PCR, another forward primer was designed and used in combination with the previously designed reverse primer. These primers proved to be specific and permitted a rapid diagnostic separation of Culicoides spp. from C. imicola.

\section{MATERIALS AND METHODS}

\subsection{Traps and collections}

We selected 19 sites at risk for the presence of $C$. imicola in (and possible introduction onto) mainland France. These sites are spread at $50 \mathrm{~km}$ intervals along the length of the French Mediterranean coast. One collection/night/site, using standard UV-light traps with a suction fan, was performed monthly from April to November 2002. The traps were set, on each night, between $1 \mathrm{~h}$ before sunset to around $8.00 \mathrm{am}$ the following morning. They were located outdoors but within $25 \mathrm{~m}$ of livestock premises and were suspended from the walls of buildings 1.5-2 $\mathrm{m}$ above the ground. A glass collecting beaker containing 200 $300 \mathrm{~mL}$ of water (this contained a drop 
Table I. Primers used for the genus-specific PCR and C. imicola specific PCR.

\begin{tabular}{lc}
\hline Primer & Sequence \\
\hline PanCulF & 5'-GTAGGTGAACCTGCGGAAGG-3 \\
PanCulR & 5'-TGCGGTCTTCATCGACCCAT-3' \\
Cul-Imicola 5'-ATTACAGTGGCTTCGGCAAG-3'
\end{tabular}

of detergent to reduce surface tension) was placed at the base of each trap. The collected insects were transported to the laboratory in the water-filled collecting beaker and then recovered and preserved in $90 \%$ ethanol. Ceratopogonidae were first separated from all other insects. Identification of $\mathrm{Culi}$ coides was initially based upon wing pattern, and confirmed subsequently by mounting a number of specimens on microscope slides $[14,27]$.

\subsection{Extraction of genomic DNA}

Single specimens of Culicoides were used for the extraction of DNA. After grinding of the insect in a mortar and pestle containing liquid nitrogen, the genomic DNA was extracted with the DNeasy Tissue kit (Qiagen, USA) according to the manufacturer's instructions.

\subsection{PCR primers}

The gene analysis software Vector NTI (Informax Inc, USA) was used to compare the ITS1 rDNA sequences of the 11 species of Culicoides retrieved from Genbank/ EMBL databases for the design of primers. Two sets of primers were defined. The first set of primers (PanCulF/PanCulR) was designed for a genus-specific PCR, the other set of primers (Cul-imicola/PanCulR) for a $C$. imicola specific PCR. Table I lists the sequences of the primers used.

\subsection{PCR amplification of ITS1}

The rDNA locus consists of one very long array of repeat units (500-700 repeats per genome). Each repeat is $9 \mathrm{~kb}$ long and consists of the genes $18 \mathrm{~S}, 5.8 \mathrm{~S}$, and $28 \mathrm{~S}$ rDNA. These genes are separated by spacers, the internal transcribed spacers (ITS1 and ITS2), and the intergenic spacer (IGS) (Fig. 1). The ITS1 of each of the eight most abundant species of Culicoides and of C. imicola was amplified by genus-specific PCR using the PanCulF/PanCulR primers and by C. imicola specific PCR using the Cul-imicola/PanCulR primers. Reactions for the PanCulF/PanCulR primers were performed in a total volume of $50 \mu \mathrm{L}$ consisting of $10 \times$ $\mathrm{PCR}$ reaction buffer, $1.5 \mathrm{mM} \mathrm{MgCl}_{2}, 250 \mu \mathrm{M}$ of each dATP, dCTP, dGTP and dTTP (Eurobio, France), $200 \mathrm{ng}$ of each primer, and 2.5 U Taq DNA polymerase under the following cycling conditions: an initial denaturation stage at $94{ }^{\circ} \mathrm{C}$ for $5 \mathrm{~min}$; then 30 cycles at $94{ }^{\circ} \mathrm{C}, 1 \mathrm{~min} ; 58{ }^{\circ} \mathrm{C}, 1 \mathrm{~min}$; $72{ }^{\circ} \mathrm{C}, 1 \mathrm{~min}$ and a final extension phase at $72{ }^{\circ} \mathrm{C}$ for $10 \mathrm{~min}$. The reaction for the Culimicola/PanCulR primers was the same as described above except for the hybridation temperature that was increased to $60{ }^{\circ} \mathrm{C}$. A volume of $1 \mu \mathrm{L}$ of genomic DNA was added to each PCR reaction and samples without DNA were included in each amplification run to exclude carry-over contamination. PCR products were examined by electrophoresis in a $1.0 \%$ agarose gel with ethidium bromide.

\section{RESULTS}

\subsection{Collection of Culicoides along coastal mainland France}

The genus Culicoides was represented by 16197 specimens belonging to 44 species, and were collected during 109 nights of sampling between April and November 2002. No specimens of $C$. imicola were found. Table II summarises the Culicoides species composition, expressed as a percentage of the total Culicoides population sampled. The species mentioned in this table are the ones representing more than $1 \%$ of the whole population. 
Table II. Number and percentage of adult Culicoides captured between April and November 2002 along coastal mainland France (19 sites/8 months/109 light trap collections).

\begin{tabular}{lccc}
\hline No. & Culicoides & Number collected & $\%$ of the total population sampled \\
\hline 1 & C. newsteadi & 11898 & 73.5 \\
2 & C. obsoletus & 1290 & 8.0 \\
3 & C. scoticus & 877 & 5.4 \\
4 & C. circumscriptus & 526 & 3.2 \\
5 & C. griseidorsum & 430 & 2.7 \\
6 & C. pulicaris & 188 & 1.2 \\
7 & C. lupicaris & 178 & 1.1 \\
8 & C. submaritimus & 173 & 1.1 \\
\hline
\end{tabular}

Table III. Genbank and NCBI accession numbers and lengths of the ITS1 sequences (base pairs) of 11 species of Culicoides.

\begin{tabular}{lcc}
\hline Culicoides & Accession number & $\begin{array}{c}\text { Length of ITS1 sequence } \\
\text { (base pairs) }\end{array}$ \\
\hline C. albicans & AJ417980 & 461 \\
C. arakawai & AJ489503 & 460 \\
C. cubitalis (= C. kibunensis) & AJ417979 & 457 \\
C. grisescens & AJ417987 & 346 \\
C. imicola & AF074019 & 316 \\
C. impunctatus & AJ417986 & 330 \\
C. maritimus & AJ417981 & 427 \\
C. nubeculosus & AJ417982 & 332 \\
C. pulicaris & AJ417983 & 469 \\
C. punctatus & AJ417984 & 463 \\
C. variipennis & $\mathrm{U} 48380$ & 347 \\
\hline
\end{tabular}

\subsection{Alignment of the ITS1rDNA of the 11 species}

The ITS1 of the 11 species of Culicoides retrieved from EMBL/Genbank databases were aligned using the gene analysis software Vector NTI. Table III lists the accession numbers of each of these sequences and their length. The alignment of the ITS1 sequences (the alignment length was $590 \mathrm{bp}$ including the gaps) revealed two highly conserved regions, between 2 and 28 bp and 564 and 591 bp, respectively (Fig. 2). These regions were used for the design of the genus specific primers but could also be useful in the future for the molecular identification of Culicoides, for studying their molecular genetics and epidemiology, and for vector systematics. The positions of the primers selected for the molecular detection of Culicoides are shown in grey-coloured boxes (Fig. 2).

\subsection{Amplification of ITS1 by genus-specific PCR}

PCR reactions using genus-specific primers (PanCulF/PanCulR) were performed on the eight most-abundant species captured along the French Mediterranean coastline 


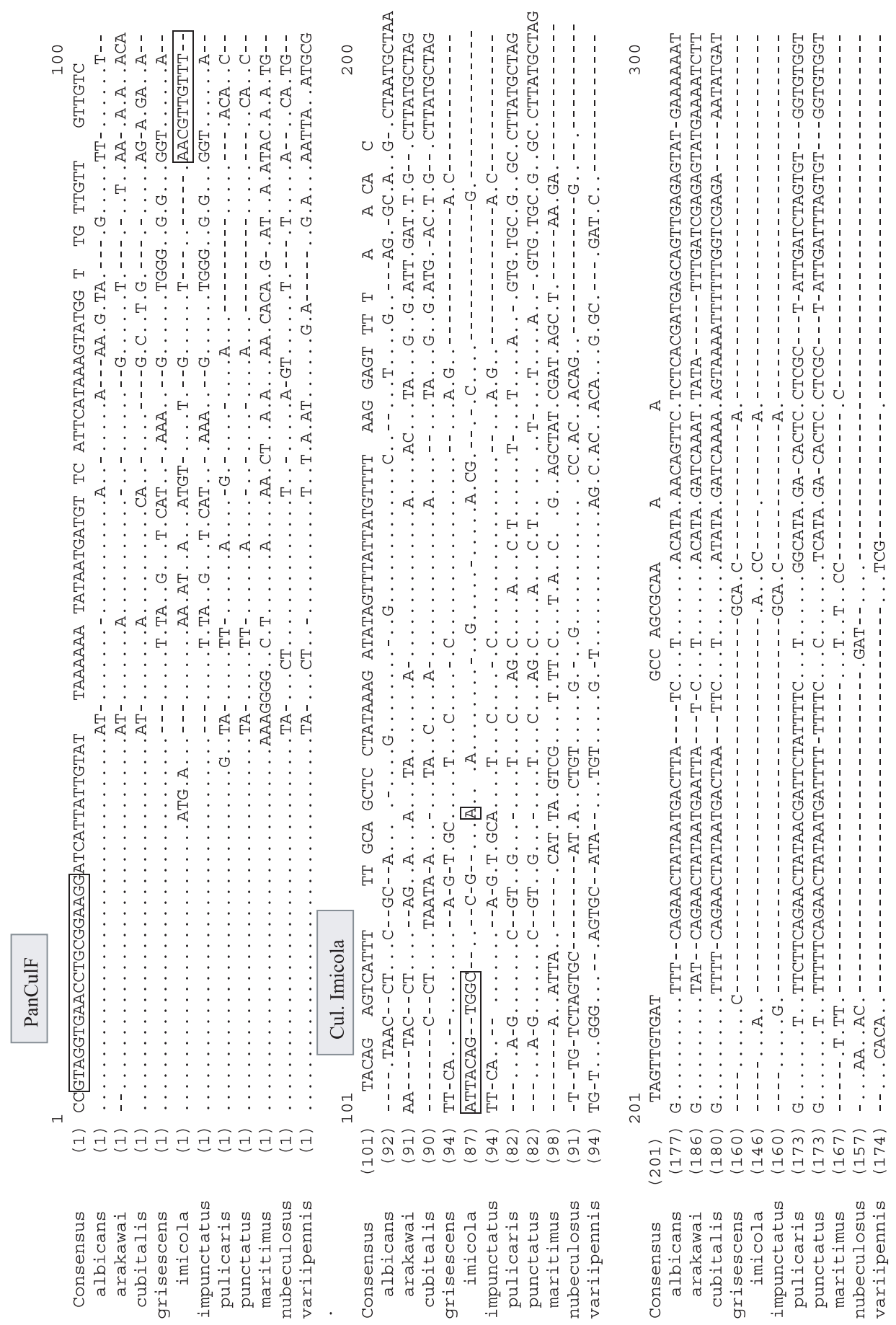


274) GCATTTCATATTATGTGT-- - - GTG.

arakawa1 (274) GCATTTTCATATTAATTGT--GTGTG.

grisescens

imicola (271) TCATTT-CATATTAAATAGAGGAGTG

impunctatus

(161)

400

punctatus

maritimus

nubeculosus

. A .

TGCCGA-GAGAATTATTGT--GTGCG

...

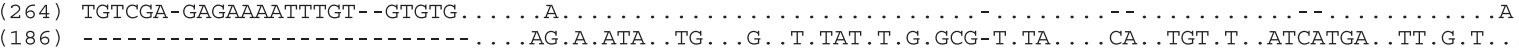

variipennis (190)

C. . W .

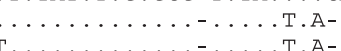

500

Consensus

TT ATTG G

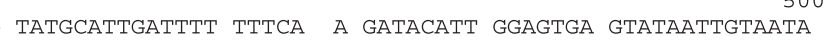

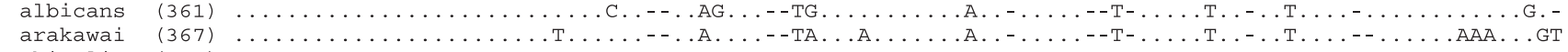

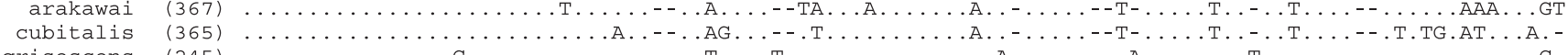

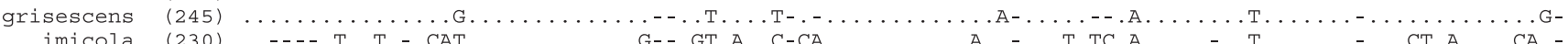

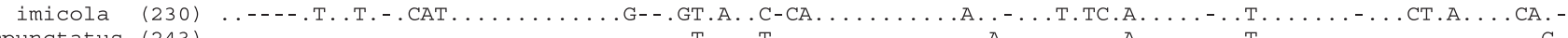

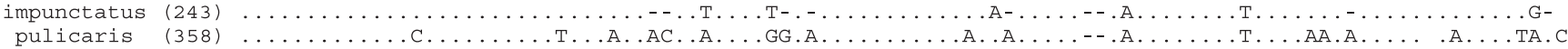

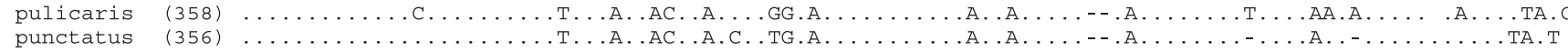

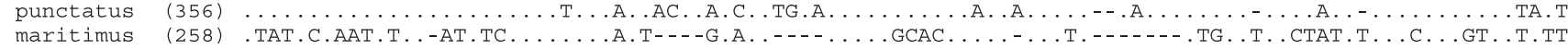

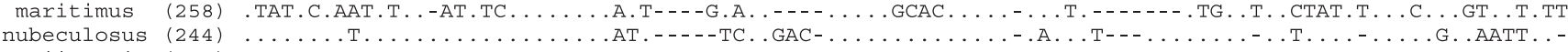

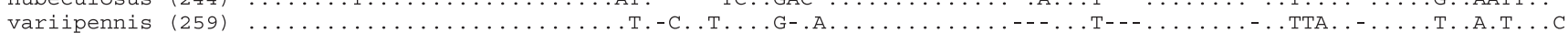

501

Consensus (501) AATT T TAAACAAAA AAAAAAAAGAT AAAAACCTTAAAC GGGGGATCA CTTGGCTCA TGGATCGATGAAGACCGCAGC

albicans

arakawai

cubitalis

grisescens

(450)

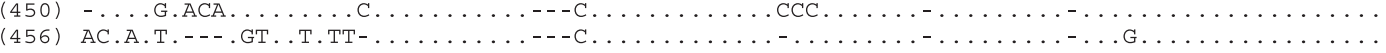

453) AC.A-_... T.A. TG-T. ........- C.

imicola

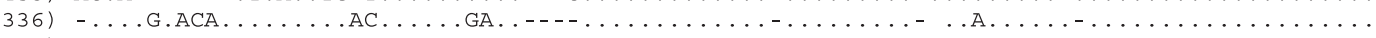

impunctatus

(318) - . . . . . . . T. T. . . . . .

GA

pulicaris

punctatus

nubeculosus

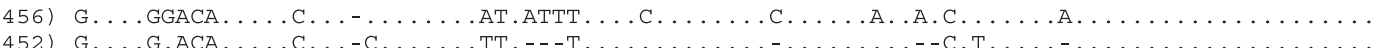

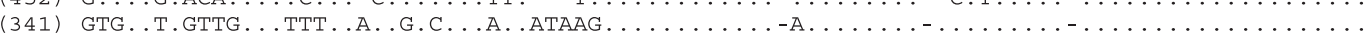

nubeculosus

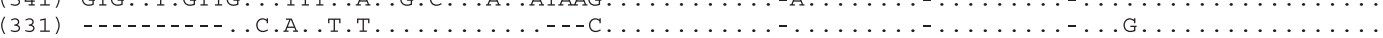

(349) АТ (-.

Figure 2. Alignment of the ITS1 of the 11 species of Culicoides known to date. In the figures, dots correspond to the conserved nucleotides. Boxed-letters indicate the sequences used to design the primers used for genusPanCulR specific PCR and the $C$. imicola-specific PCR. Block letters are the nucleotides that differ between species. 


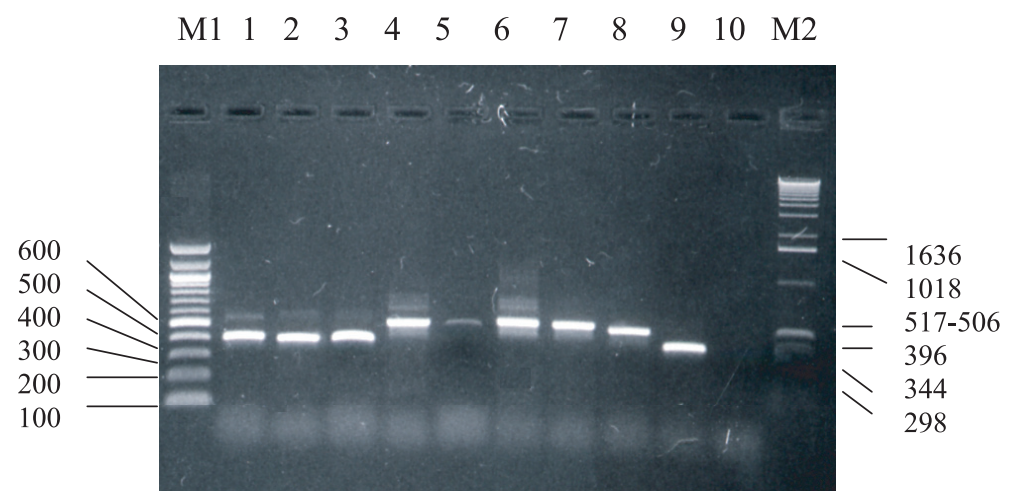

Figure 3. Genus amplification of the 9 Culicoides species ITS1 by PCR (PanCulF/PanCulR). M1 represents $100 \mathrm{bp}$ DNA ladder, M2 marker X (Roche, USA). Lanes 1 to 10 correspond respectively to C. newsteadi, C. obsoletus, C. scoticus, C. circumscriptus, C. griseidorsum, C. pulicaris, C. lupicaris, C. submaritimus, C. imicola, Negative Control.

and reference $C$. imicola specimens collected in Corsica in 2001, $150 \mathrm{~km}$ distant from southern France. Agarose gel electrophoresis (Fig. 3) showed that the genus-specific amplified PCR products of the nine Culicoides species tested (C. newsteadi, C. obsoletus, C. scoticus, C. circumscriptus, C. griseidorsum, C. pulicaris, C. lupicaris, C. submaritimus and C. imicola) occurred between 316 and 500 bp (when compared against the $100 \mathrm{bp}$ DNA ladder marker).

As expected, no band was detected in the negative control. The PCR was also performed on other species of Culicoides less commonly found on the French Mediterranean mainland (C. sahariensis, $C$. kibunensis, $C$. maritimus, $C$. punctatus, etc.). The results confirmed that the sequences targeted by our primers are highly conserved (data not shown).

\subsection{Molecular identification of C. imicola by specific ITS1 PCR}

PCR reactions were performed in order to evaluate the specificity of $C$. imicola primers (Cul-imicola/PanCulR) for C. imicola, compared to other Culicoides species. Agarose gel electrophoresis (Fig. 4) showed no specific PCR products for each of the eight non- $C$. imicola ITS1 species tested (C. newsteadi, C. obsoletus, C. scoticus, $C$. circumscriptus, C. griseidorsum, C. pulicaris, C. lupicaris, and C. submaritimus). However, a specific band (303 bp) was amplified for $C$. imicola. Again, no band was detected in the negative control. Thus, we developed a specific tool for the detection of $C$. imicola.

\subsection{Specificity of the PCR assay}

Because surveillance activities for most vector species are performed using pooled genus specimens, we wished to evaluate whether our specific PCR assay was useful to detect $C$. imicola within a pool of other biting midges. Agarose gel electrophoresis (Fig. 5) showed that no specific PCR products were amplified in lane 1, corresponding to a pool of one specimen of each of the eight most-abundant Culicoides species (C.newsteadi, C. obsoletus, C. scoticus, C. circumscriptus, C. griseidorsum, C. pulicaris, $C$. lupicaris, $C$. submaritimus). The addition of one or ten specimens of $C$. imicola (lanes 2 and 3 respectively) to this pool yielded the expected band of $303 \mathrm{bp}$ following PCR amplification. Again, no band was 


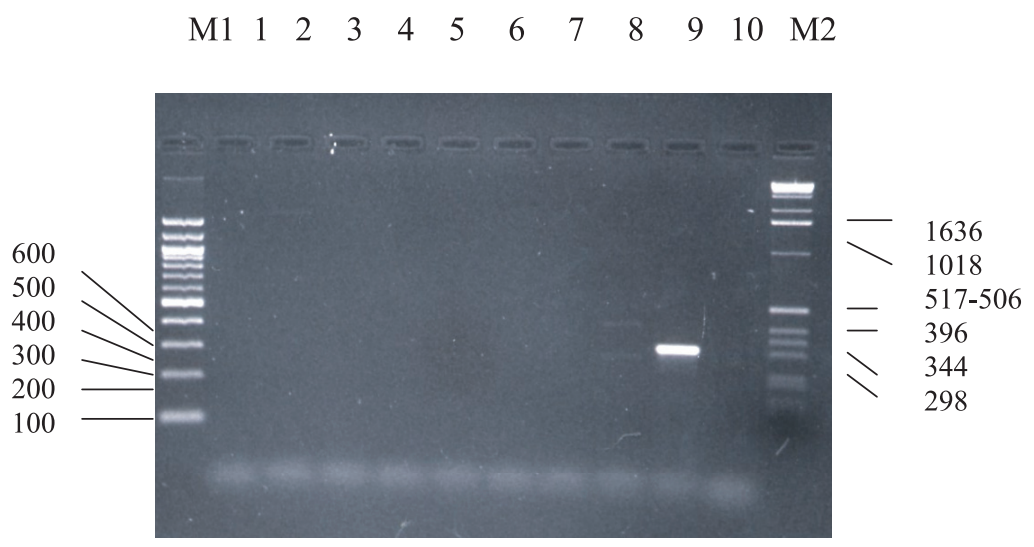

Figure 4. Amplification of the 9 Culicoides species ITS1 by specific PCR (Cul-Imicola/PanCulR). M1 represents 100 bp DNA ladder, M2 marker X (Roche, USA). Lanes 1 to 10 correspond respectively to $C$. newsteadi, C. obsoletus, C. scoticus, C. circumscriptus, C. griseidorsum, C. pulicaris, C. lupicaris, C. submaritimus, C. imicola, Negative Control.

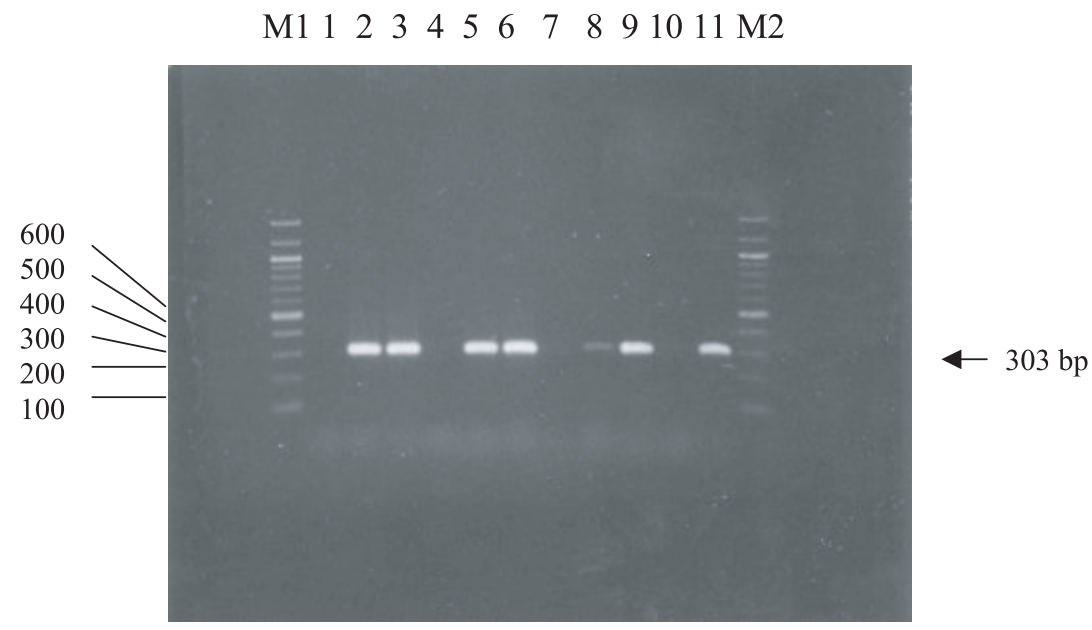

Figure 5. The specificity of the Culicoides imicola specific PCR amplification (Cul-Imicola/ PanCulR). M1 and M2 represent 100 bp DNA ladder. Lane 1 corresponds to a mix of 1 specimen of each of the eight most abundant species C. newsteadi, C. obsoletus, C. scoticus, C. circumscriptus, C. griseidorsum, C. pulicaris, C. lupicaris, C. submaritimus. Lanes 2 and 3 are equivalent to lane 1 with one and 10 specimens of $C$. imicola respectively. Lane 4 corresponds to ten specimens of Forcipomyia spp. Lanes 5 and 6 correspond to lane 4 with one and 10 specimens of $C$. imicola respectively. Lane 7 corresponds to a pool of different Diptera families (Psychodidae, Chironomidae, Culicidae, Cecidomyidae, Sciaridae, Tipulidae) and other orders of insects (Lepidoptera, Coleoptera, Hymenoptera, Homoptera) recovered from a light-trap. Lanes 8 and 9 correspond to lane 7 plus one and 10 specimens of $C$. imicola respectively. Lane 10 is the negative control. Lane 11 is a $C$. imicola positive control. 

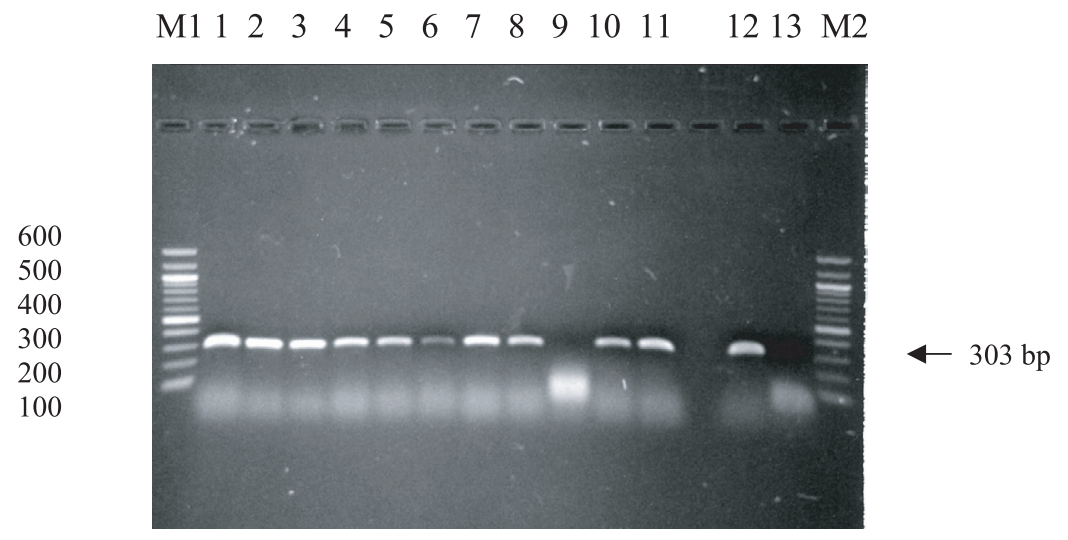

Figure 6. Sensitivity of the Culicoides imicola specific PCR amplification (PanCulR/Cul-Imicola). M1 and M2 represent $100 \mathrm{bp}$ DNA ladder. Lanes 1 to 8 correspond respectively to one specimen of $C$. imicola in 25, 50,100, 200, 400, 800, 1600 and 3200 specimens of C. newsteadi. Lane 9 corresponds to $6.5 \mathrm{mg}$ of dried materials recovered from a light trap without any Culicoides specimens. Lanes 10 and 11 correspond to lane 9 with one and 10 specimens of $C$. imicola respectively. Lane 11 corresponds to lane 9 with ten specimens of $C$. imicola. Lanes 12 and 13 correspond respectively to the positive and negative controls.

detected (lane 4) following PCR on a pool of 10 specimens of Forcipomyia spp., a genus belonging to the Ceratopogonidae family. However, a specific band was amplified when one or ten specimens of C. imicola (lanes 5 and 6 respectively) were added to the pool. Finally, a mixture of different insect families recovered from a light-trap equivalent to $6.5 \mathrm{mg}$ of dried materials (lane 7) did not result in the amplification of specific DNA, but when one or ten specimens of $C$. imicola were added (lanes 8 and 9, respectively), amplification of a specific band resulted.

\subsection{Sensitivity of the PCR assay}

In order to assess the sensitivity of the technique, C. imicola-specific PCR reactions were performed on $C$. imicola within a range of pooled specimen sizes. Figure 6 shows agarose gel electrophoresis of specific PCR products that were amplified in serial samples containing one specimen of C. imicola in 25, 50, 100, 200, 400, 800, 1600 and 3200 specimens of C. newsteadi (lanes 1 to 8 respectively). Lane 9 corresponds to $6.5 \mathrm{mg}$ of dried material recovered from a light trap without any Culicoides specimens, showing that no band was detected. Finally, when one or ten specimens of $C$. imicola were added to the dried material pool from the trap, a specific band was amplified (lanes 10 and 11). As expected, no band was detected in the negative control (lane 13).

\section{DISCUSSION}

In its recent re-emergence in the Mediterranean Basin, and particularly in Corsica in 2000 and 2001, it is believed that BTV was transmitted principally by the classical Afro-Asiatic insect vector C. imicola. However, in the eastern Mediterranean Basin, outbreaks of the disease have occurred in Bulgaria, Serbia, Kosovo, Croatia, Montenegro, northern Greece and in Bosnia Herzegovina where $C$. imicola has not been detected during insect surveys. Recently, the virus was isolated from Culicoides represented by one or more species of the 
C. obsoletus complex on mainland Italy [24] and C. pulicaris on the island of Sicily [5].

C. imicola is also the vector for the African horse sickness, another orbiviral disease that can be especially devastating to equids. The monitoring of Culicoides species is therefore essential in areas at risk for the management of animal health. Since the biology of C. imicola and its persistence in a given biotope appear to be largely influenced by climatic conditions, the spectre of possible global climate change suggests that monitoring efforts should be sustained over a long period of time. This is particularly true in areas like southern France where trapping frequencies will be increased to sample high quantities of insects for an early detection of $C$. imicola. However, in such extensive monitoring programmes the morphological identification of Culicoides has been limited to the labour-intensive and time consuming techniques of morphological taxonomy, species prevalence and geographic distribution. This is the reason why we decided to investigate the possibility to detect Culicoides spp. and C. imicola using molecular tools. From the molecular point of view, recent studies on this genus hold promise for the development of tools that will lead to the rapid and reliable identification of C. imicola and other potential vectors of BTV [15]. The nuclear ribosomal DNA arrays of eukaryotic organisms usually consist of tandemly repeated copies of the transcriptional unit and non-transcribed spacer, the rDNA transcriptional unit consisting of three gene regions (i.e. $18 \mathrm{~S}, 5.8 \mathrm{~S}$ and 28S) separated by transcribed spacers [8]. As shown in Figure 1, the external transcribed spacer (ETS) is located upstream of the 18S gene, with the first (ITS-1) and second (ITS-2) internal transcribed spacers (ITS) located between either genes $18 \mathrm{~S}$ and $5.8 \mathrm{~S}$, or between $5.8 \mathrm{~S}$ and $28 \mathrm{~S}$, respectively. The rDNA has several unique features which make it an ideal target for use in DNA species-diagnostic assays [6]. It contains regions of varying evolutionary rates, from highly conserved regions (e.g. 5.8S and 18S rRNA genes) to more variable ones (e.g. spacers). Species from other genera have been studied also showing that rDNA provides genetic markers for the identification of mosquitoes (Anopheles gambiae, An. nili and An. minimus using ITS) $[9,10,13,22,26]$. In another study the ITS1 sequences of $C$. arakawai, and eight other species, were used to produce an initial partial phylogeny of the species within the genus [15].

Thus, our objective in this study was to establish a diagnostic test for the rapid detection of the main BTV-transmitting species, $C$. imicola. Even if the ITS1 sequences of most of the species present in the Mediterranean Basin remain unknown, the choice of the primers in a highly conserved region amongst sequenced species, should serve for the detection of the majority of Culicoides species. After genus PCR reaction, the nine Culicoides species (including $C$. imicola), yielded an amplified fragment of 316 to $500 \mathrm{bp}$. Other Culicoides species could also be detected by these primers (data not shown), thus demonstrating the validity of our initial hypothesis. Interestingly, the highly variable size of the amplified products (up to 180 bases) illustrates that ITS-1 may differ widely between species of the same genus. This reinforces the potential interest of ITS-1 for phylogenetic studies of Culicoides. We are now sequencing all our PCR products with the objective of performing a phylogenetic analysis.

The specific $C$. imicola primer was designed to amplify in a more variable region where numerous substitutions or deletions were present. The primer that was selected proved to be specific for $C$. imicola. Because two forward primers combined with a unique reverse primer provides resolution at the genus and species levels, development of a useful multiplex PCR should be possible. We are now evaluating this possibility as well as developing a real-time PCR test as a faster and quantitative diagnostic tool.

When we evaluated the sensitivity of our PCR, we found that only one specimen of each Culicoides was sufficient to obtain a 
PCR product. In addition, our PCR was able to specifically detect one $C$. imicola within 3200 other Culicoides (Fig. 6) or within $6.5 \mathrm{mg}$ of dried materials collected from a light trap. That amount of material $(6.5 \mathrm{mg})$ represents approximately a third of the insect mass collected by a light trap during the maximum Culicoides activity period in France. Interestingly, the sensitivity of our PCR was greatly improved when the genomic DNA was diluted by 10 before amplification. The robust nature of this technique for C. imicola surveillance is also demonstrated by its ability to specifically detect one specimen of C. imicola within 8 other Culicoides species, within 10 specimens of Forcipomyia spp., or within a pool of different Diptera families and other orders of insects.

Another potential application of this work will be to adapt this technique to Culicoides larvae, in order to help in the identification of the larvae and then in the characterisation of their morphological differences and also to define the larvae biotope of $C$. imicola and the biological parameters which are involved in their development (duration, diapause, aestivation).

In conclusion, we developed a rapid and qualitative diagnostic tool for Culicoides spp. and C. imicola that should be useful for routine epidemiological and surveillance monitoring of this orbivirus vector. Our work also suggests that specific forward primers for other Culicoides species that are vectors for bluetongue or African horse sickness virus could be designed and used as well.

\section{REFERENCES}

[1] Baylis M., The re-emergence of bluetongue, Vet. J. 164 (2002) 5-6.

[2] Baylis M., Mellor P.S., Bluetongue around the Mediterranean in 2001, Vet. Rec. 149 (2001) 659.

[3] Borkent A., Wirth W.W., World species of biting midges (Diptera: Ceratopogonidae), Bulletin of the American Museum of Natural History No. 233, 257 p.
[4] Capela R., Purse B.V., Pena I., Wittman E.J., Margarita Y., Capela M., Romao L., Mellor P.S., Baylis M., Spatial distribution of Culicoides species in Portugal in relation to the transmission of African horse sickness and bluetongue viruses, Med. Vet. Entomol. 17 (2003) 165-177.

[5] Caracappa S., Torina A., Guercio A., Vitale F., Calabro A., Purpari G., Ferrantelli V., Vitale M., Mellor P.S., Identification of a novel bluetongue virus vector species of $\mathrm{Culi}$ coides in Sicily, Vet. Rec. 153 (2003) 71-74.

[6] Collins F.H., Paskewitz S.M., A review of the use of ribosomal DNA (rDNA) to differentiate among cryptic Anopheles species, Insect. Mol. Biol. 5 (1996) 1-9.

[7] Delécolle J.C., de La Rocque S., Contribution à l'étude des Culicoides de Corse. Liste des espèces recensées en 2000/2001 et redescription du principal vecteur de la fièvre catarrhale ovine: C. imicola Kieffer, 1913 (Diptera: Ceratopogonidae), Bull. Soc. Entomol. Fr. 107 (2002) 371-379.

[8] Elder J.F., Turner B.J., Concerted evolution of repetitive DNA sequences in eukaryotes, Q. Rev. Biol. 70 (1995) 297-320.

[9] Gentile G., Slotman M., Ketmaier V., Powell J.R., Caccone A., Attempts to molecularly distinguish cryptic taxa in Anopheles gambiae s.s, Insect. Mol. Biol. 10 (2001) 25-32.

[10] Gentile G., Della Torre A., Maegga B., Powell J.R., Caccone A., Genetic differentiation in the African malaria vector, Anopheles gambiae s.s., and the problem of taxonomic status, Genetics 161 (2002) 1561-1578.

[11] Holmes I.H., Boccardo G., Estes M.K., Furuichi M.K., Family Reoviridae, in: Murphy F.A., Fauquet C.M., Bishop D.H.L., Ghabrial S.A. (Eds.), Virus Taxonomy. Classification and nomenclature of viruses. Sixth report of the international committee on taxonomy of viruses. Springer, Wien, New York, Arch. Virol. Suppl. 10 (1995) 208-239.

[12] Howell P., The 1960 epizootic of AHS in the middle East and SW Asia, J. South African Vet. Med. Assoc. 31 (1960) 329-334.

[13] Kengne P., Awono-Ambene P., AntonioNkondjio C., Simard F., Fontenille D., Molecular identification of the Anopheles nili group of African malaria vectors, Med. Vet. Entomol. 17 (2003) 67-74.

[14] Kremer M., Contribution à l'étude du genre Culicoides Latreille particulièrement en France, Encyclop. Ent. Série A, Ed. P. Lechevallier, Paris, 1965, 299 p. 
[15] Li G.Q., Hu Y.L., Kanu S., Zhu X.Q., PCR amplification and sequencing of ITS1 rDNA of Culicoides arakawai, Vet. Parasitol. 112 (2003) 101-108.

[16] Linto Y.M., Mordue Luntz A.J., Cruickshank R.H., Meiswinkel R., Mellor P.S., Dallas J.F., Phylogenetic analysis of the mitochondrial cytochrome oxidase subunit I gene of five species of the Culicoides imicola species complex, Med. Vet. Entomol. 16 (2002) 139-146.

[17] Meiswinkel R., Afrotropical Culicoides: a redescription of $C$. (Avaritia) imicola Kieffer, 1913 (Diptera: Ceratopogonidae) with description of the closely allied $C$. (A.) bolitinos $\mathrm{sp}$. nov. reared from the dung of the African buffalo, blue wildebeest and cattle in South Africa, Onderstepoort J. Vet. Res. 56 (1989) 23-39.

[18] Mellor P.S., The replication of bluetongue virus in Culicoides vectors, Curr. Top. Microbiol. Immunol. 162 (1990) 143-161.

[19] Mellor P.S., Wittmann E.J., Bluetongue virus in the Mediterranean Basin 1998-2001, Vet. J. 164 (2002) 20-37.

[20] Mellor P.S., Boorman J., Baylis M., Culicoides biting midges: their role as arbovirus vectors, Annu. Rev. Entomol. 45 (2000) 307340.

[21] Miranda M.A., Borras D., Rincon C., Alemany A., Presence in the Balearic Islands (Spain) of the midges Culicoides imicola and Culicoides obsoletus group, Med. Vet. Entomol. 17 (2003) 52-54.

[22] Paskewitz S.M., Ng K., Coetzee M., Hunt R.H., Evaluation of the polymerase chain reaction method for identifying members of the Anopheles gambiae (Diptera: Culicidae) complex in southern Africa, J. Med. Entomol. 30 (1993) 953-957.

[23] Sarto i Monteys V., Saiz-Ardanaz M., Culicoides midges in Catalonia (Spain), with special reference to likely bluetongue virus vectors, Med. Vet. Entomol. 17 (2003) 288-293.
[24] Savini G., Goffredo M., Monaco F., de Santis P., Meiswinkel R., Transmission of bluetongue virus in Italy, Vet. Rec. 152 (2003) 119.

[25] Takamatsu H., Mellor P.S., Mertens P.P., Kirkham P.A., Burroughs J.N., Parkhouse R.M., A possible overwintering mechanism for bluetongue virus in the absence of the insect vector, J. Gen. Virol. 84 (2003) 227 235.

[26] Van Bortel W., Trung H.D., Roelants P., Harbach R.E., Backeljau T., Coosemans M., Molecular identification of Anopheles minimus s.l. beyond distinguishing the members of the species complex, Insect. Mol. Biol. 9 (2000) 335-340.

[27] Wirth W.W., Marston N., A method for mounting small insects on microscope slides in canada balsam, Ann. Entomol. Soc. Am. 61 (1968) 783-784.

[28] Wittmann E.J., Mellor P.S., Baylis M., Using climate data to map the potential distribution of Culicoides imicola (Diptera: Ceratopogonidae) in Europe, Rev. Sci. Tech. 20 (2001) 731-740.

[29] Zientara S., De la Rocque S., Gourreau J.M., Gregory M., Diallo A., Hendricks P., Libeau G., Sailleau C., Delécolle J.C., La fièvre catarrhale ovine en Corse en 2000, Épidémiologie et santé animale 38 (2000) 133-144.

[30] Zientara S., Grillet C., De la Rocque S., Gourreau J.M., Gregory M., Diallo A., Hendricks P., Libeau G., Sailleau C., Albina E., Breard E., Delécolle J.C., La fièvre catarrhale ovine en Corse en 2001, Épidémiologie et santé animale 40 (2001) 129-137.

[31] Zientara S., Sailleau C., Dauphin G., Roquier C., Remond E.M., Lebreton F., Hammoumi S., Dubois E., Agier C., Merle G., Breard E., Identification of bluetongue virus serotype 2 (Corsican strain) by reverse-transcriptase PCR reaction analysis of segment 2 of the genome, Vet. Rec. 150 (2002) 598-601. 